\title{
SENSORIAMENTO REMOTO MULTIFONTE APLICADO AO ESTUDO DO CORPO HÍDRICO DO RIO JULIANA NA APA DO PRATIGI
}

\author{
$\underline{\text { Erli Pinto dos Santos }}{ }^{1}$; Taíse Bomfim de Jesus ${ }^{2}$ e Ayala de Souza Reis Carneiro ${ }^{3}$ \\ 1. Bolsista PIBIC/CNPq, Engenheiro Agrônomo, Universidade Estadual de Feira de Santana, mestrando em \\ Engenharia Agrícola, bolsista CNPq, Universidade Federal de Viçosa, e-mail: erlitec.agri@ hotmail.com \\ 2. Orientador, Departamento de Ciências Exatas, Universidade Estadual de Feira de Santana, e-mail: \\ taisebj@hotmail.com \\ 3. Participante do projeto de pesquisa do Orientador, Departamento de Ciências Biológicas, Universidade Estadual de \\ Feira de Santana, e-mail: ayala.reis@ @otnail.com
}

PALAVRAS-CHAVE: Espectrorradiometria; Sentinel-2; Rio Juliana.

\section{INTRODUÇÃO}

A redução da qualidade das águas dos rios, reservatórios, estuários e regiões costeiras, é uma preocupação mundial visto que resulta na restrição de seus múltiplos usos (CICERELLI; GALO, 2015) e nessa ótica a pesquisa tem analisado diversas substâncias e seres vivos presentes na água na busca de identificar padrões de qualidade para monitorar a presença destes (FERREIRA; GALO, 2012), dentre estes a clorofila-a tem sido muito estudada por ser um componente opticamente ativo (COA) em sensores. E nesse contexto se insere o sensoriamento remoto como ferramenta eficaz no monitoramento da qualidade da água, pois recobre extensas áreas e permite uma análise espaço-temporal, onde nos dados é permitido inferir sobre a presença de COAs.

Dessa forma o objetivo deste trabalho é analisar as variáveis (espectral e limnológica) do corpo hídrico no rio Juliana, na Área de Preservação Ambiental (APA) do Pratigi, correlacionando os dados de espectrorradiometria de campo e reflectância por satélite com a concentração da clorofila-a.

\section{METODOLOGIA}

A área de estudo, ilustrada na figura 1, compreende um lago artificial localizado no curso do rio Juliana, na Bacia Hidrográfica do Rio Juliana, na APA do Pratigi. A sua escolha se deu pela facilidade de acesso, pois, encontra-se dentro da Fazenda Juliana, além de o lago apresentar espelho d'água maior que 40 ha, dimensão que permite uso de imagem de satélite no seu estudo. Os pontos amostrais usados são os de 1 a 4 , como visto na figura 1. A clorofila-a, variável limnológica escolhida para caracterizar o corpo hídrico em estudo, foi quantificada usando o método do Standard Methods for Water and Wastwater Examination e o material para análise foi coletado no campo realizado no dia 28/11/2017, onde concomitantemente os dados espectrorradiométricos foram coletados usando um espectrorradiômetro FieldSpec 4 Hi-Res de acordo com metodologia aplicada por Santos, Carneiro e Jesus (2017): na coleta há a mensuração da reflectância, sendo de interesse os valores na região espectral fotossinteticamente ativa (de 400 a $900 \mathrm{~nm}$ ). Nos dados espectrorradiométricos foi aplicada a primeira derivada como técnica de extração de informações, seguindo a metodologia aplicada por Santos, Carneiro e Jesus (2017). 
Figura 1. Lago na Bacia Hidrográfica do Rio Juliana na APA do Pratigi usada como área de estudo.

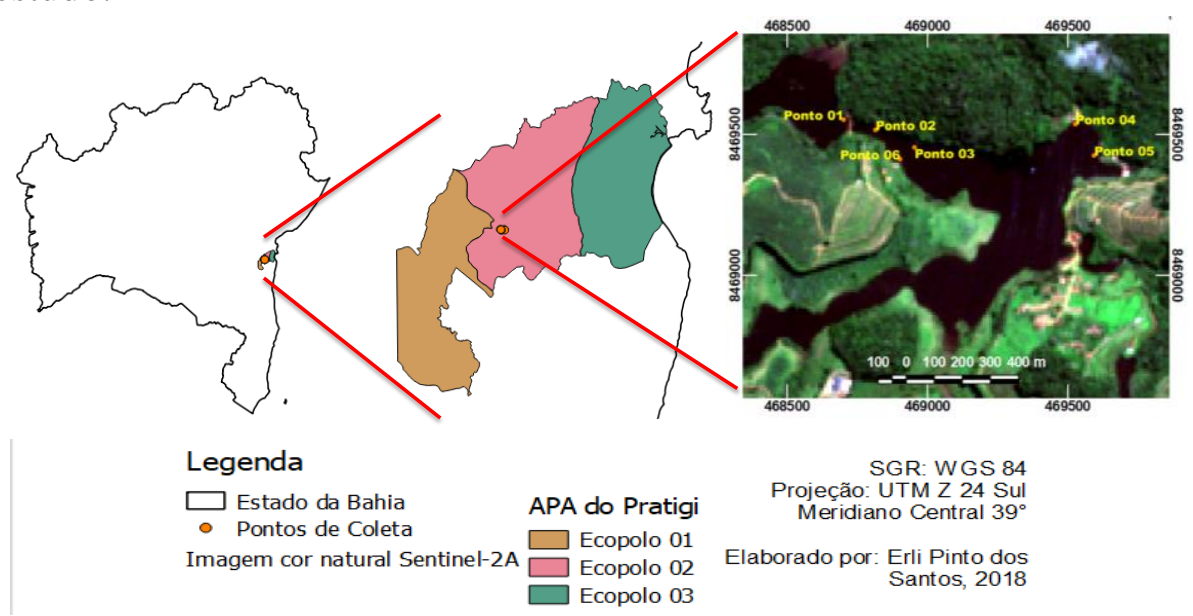

Além dos dados acima mencionados foi coletada a partir do portal Copernicus Open Access Hub (https://scihub.copernicus.eu) uma imagem do sensor MSI (Multispectral Sensor) do satélite Sentinel-2A, cuja medição data de 27/11/2017. A imagem foi utilizada para calcular a matemática de bandas (equação 1) usada por Toming et al. (2016) para relacionar com a concentração de clorofila-a, usando a reflectância das bandas B06, B05 e B04 do MSI (Infravermelho limítrofe 2, limítrofe 1 e vermelho) com e sem correção atmosférica (reflectância na base da atmosfera (BOA) e no topo da atmosfera (TOA), respectivamente), sendo que a correção atmosférica foi realizada com o modelo proposto por European Space Agency (2016) baseada na transferência radiativa libRadtran (Mayer e Kylling, 2005).

$$
B 05-\frac{B 04+B 06}{2}
$$

Então para melhor visualização de todos os dados e verificar relação das concentrações de clorofila-a com a radiação eletromagnética em dados multi e hiperespectrais, foi aplicada a técnica de correlação de variáveis aleatórias e a análise de regressão linear pelo método dos mínimos quadrados para analisar o comportamento da variável limnológica com a variável espectral.

\section{RESULTADOS E DISCUSSÃO}

O primeiro resultado trata-se da determinação das quantidades de clorofila-a das amostras dos pontos de estudo, exibidas na tabela 1. A clorofila-a é uma variável limnológica largamente aplicada nos estudos com COAs nos trabalhos de observação da Terra, pois ela denota a presença de biomassa de fitoplânctons nos corpos hídricos indica o estado trófico, e é essencial para interpretação de dados de sensoriamento remoto (GITELSON; SCHALLES; HLADIK, 2007; TOMING et al., 2016; HA et al., 2017) pois sua quantificação é um método analítico estabelecido.

Tabela 1. Valores de clorofila-a encontrados para as amostras dos pontos.

\begin{tabular}{l|l}
\hline Ponto amostral & Concentração de \\
\hline
\end{tabular}




\begin{tabular}{l|c}
\hline & clorofila- $\boldsymbol{a}(\boldsymbol{\mu g} / \boldsymbol{L})$ \\
\hline $\mathbf{0 1}$ & 28,74 \\
$\mathbf{0 2}$ & 26,13 \\
$\mathbf{0 3}$ & 12,02 \\
$\mathbf{0 4}$ & 16,21 \\
\hline
\end{tabular}

Uma das formas de interpretar dados espaciais obtidos por sensores e variáveis ambientais é a análise multivariada, que permite observar o comportamento de duas variáveis independentes, portanto correlação foi aplicada para verificar o quanto a clorofila-a se relaciona com os dados dos sensores orbitais e de campo, bem como a regressão linear foi usada para verificar a feição dessa relação: estes resultados encontram-se na figura $2 \log$ abaixo.

Figura 2. A e B: correlação entre a altura do pico de $705 \mathrm{~nm}$ calculada a partir dos dados do Sentinel-2 e a concentração de clorofila-a, com dados oriundos da reflectância no topo da atmosfera na base da atmosfera, respectivamente; C: Correlação entre a reflectância em $672 \mathrm{~nm}$ (região do espectro vermelho) e a concentração de clorofila-a; D: Correlação entre a primeira derivada em $670 \mathrm{~nm}$ (região do espectro vermelho) e a concentração de clorofila-a.
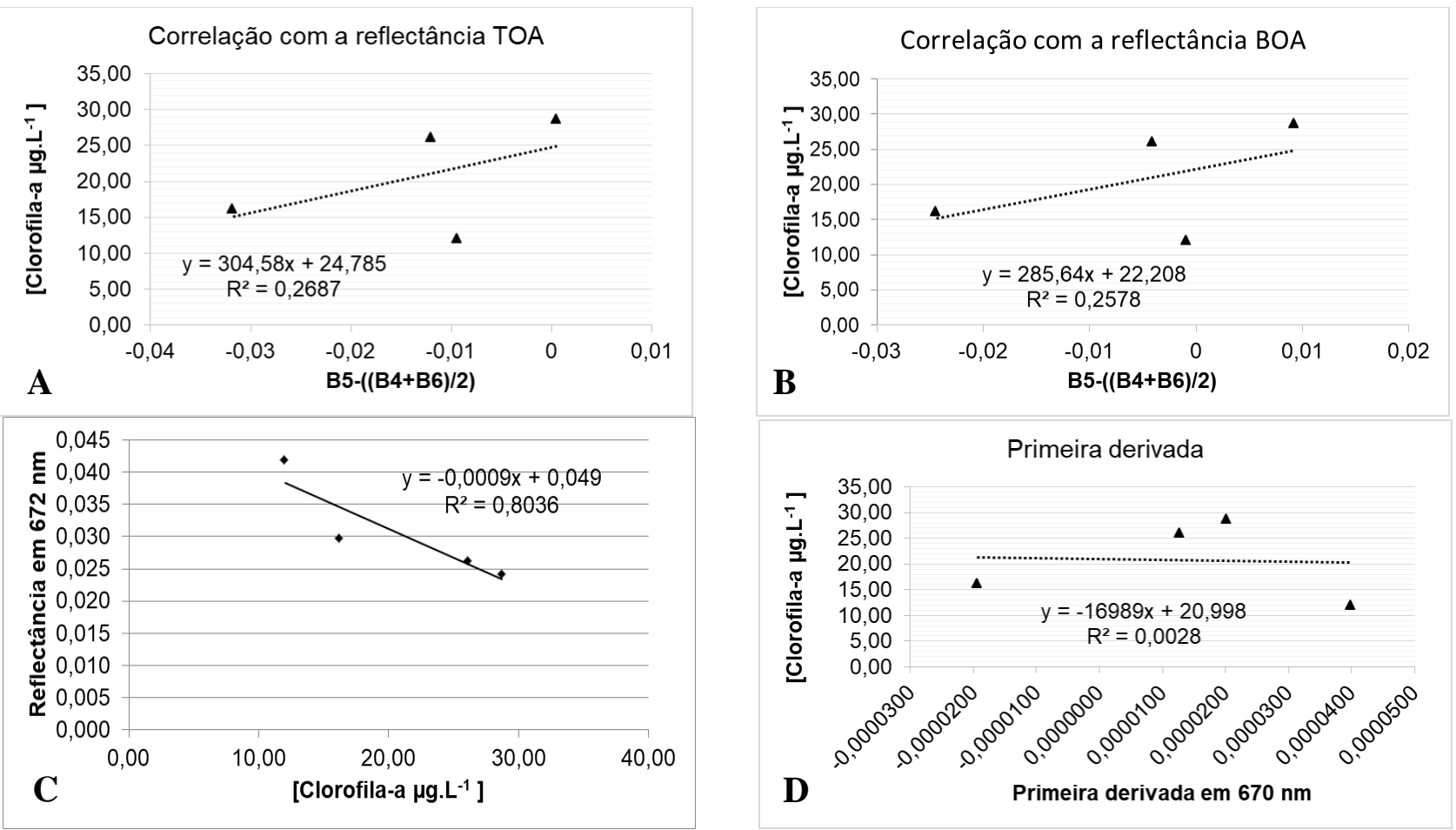

Há maior correlação com os dados oriundos da reflectância TOA do que com a reflectância BOA (figuras 2 A e B), além disso observa-se que a linha de tendência é crescente, corroborando com Toming et al., (2016), entretanto os autores obtiveram coeficientes de correlação maiores do que os das figuras $2 \mathrm{~A} \mathrm{e} \mathrm{B}$, pois, para reflectância TOA e BOA, encontraram correlações da ordem de 0,83 e 0,80 . Isso pode ser explicado pelo número de amostras representativas tomadas no trabalho: Toming et al. (2016) obtiveram a clorofila-a de 22 amostras, e neste trabalho apenas 4. Mas apesar de as correlações serem menores, guardam a relação de os dados de reflectância TOA serem 
superiores à reflectância BOA. A melhor correlação com a reflectância espectral (figura 2 C) encontrada neste trabalho foi obtida na região de $675 \mathrm{~nm}\left(\mathrm{r}^{2}=0,80\right)$, região onde Rundquist et al., (1996) e Gitelson (1992) obtiveram picos de absorbância na região do vermelho associando à concentração da clorofila-a, quanto menor a reflectância nesse pico maior a concentração de clorofila, e isso foi observado. Contudo, a mesma correlação não pôde ser observada confrontando os dados de primeira derivada (figura 2 D), pois, foram escolhidos os valores de primeira derivada no comprimento de onda de $670 \mathrm{~nm}$, onde Rundquist et al. (1996) obtiveram valores de $\mathrm{r}^{2}$ entre 0,90 e 0,93, e neste trabalho pode-se dizer que não há correlação entre as duas variáveis, uma vez que o coeficiente de correlação encontrado foi de 0,0028 .

\section{CONCLUSÃO}

Foi possível correlacionar os dados de clorofila-a com a reflectância por satélite e por espectrorradiometria de campo, além da derivada espectral, onde a espectrorradiometria em $672 \mathrm{~nm}$ apresentou melhor correlação; há um potencial de uso do sensor MSI/Sentinel-2 desde que aumentada a amostragem em campo; a primeira derivada não apresentou correlação. Portanto há um potencial no uso de métodos de sensoriamento remoto, pois estes confrontam com dados químicos.

\section{REFERÊNCIAS}

SANTOS, E.P.; CARNEIRO, A.S.R.; JESUS, T.B.. Derivada espectral como técnica de extração de informações de espectros de reflectância de corpo hídrico do baixo curso do Rio Subaé, Bahia. In: SIMPÓSIO BRASILEIRO DE SENSORIAMENTO REMOTO, 18., 2017, Santos. Anais... . Santos: Inpe, 2017. p. 3894 - 3901.

HA, N.T.T.; THAO, N.T.P.; KOIKE, K.; NHUAN, M.T. 2017. Selecting the Best Band Ratio to Estimate Chlorophyll-a Concentration in a Tropical Freshwater Lake Using Sentinel 2A Images from a Case Study of Lake Ba Be (Northern Vietnam). ISPRS International Journal of Geo-information, v. 6, n. 9, p.290305, 13 set. 2017. http://dx.doi.org/10.3390/ijgi6090290.

GITELSON, A.A.; SCHALLES, J.F.; HLADIK, C.M. 2007. Remote chlorophyll-a retrieval in turbid, productive estuaries: Chesapeake Bay case study. Remote Sensing of Environment, v. 109, n. 4, p.464472. http://dx.doi.org/10.1016/j.rse.

TOMING, K.; KUTSER, T.; LAAS, A.; SEPP, M.; PAAVEL, B.; NÕGE, T. 2016. First Experiences in Mapping Lake Water Quality Parameters with Sentinel-2 MSI Imagery. Remote Sensing, v. 8, n. 8, p.640-654. http://dx.doi.org/10.3390/rs8080640.

MAYER, B. \& KYLLING, A. 2005. Technical note: The libRadtran software package for radiative transfer calculations - description and examples of use. Atmospheric Chemistry and Physics, 5 (7): 1855 - 1877.

EUROPEAN SPACE AGENCY. 2016. Sen2Cor Configuration and User Manual. Disponível em: < http://step.esa.int/thirdparties/sen2cor/2.3.0/[L2A-SUM]\%20S2-PDGS-MPC-L2A-SUM\%20[2.3.0].pdf >. Acesso em: 15 mar. 2017.

RUNDQUIST, D. C.; HAN, L.; SCHALLES, J. F.; PEAKE, J. S. 1996. Remote Measurement of Algal Chlorophyll in Surface Waters: The Case for the First Derivative of Reflectance Near $690 \mathrm{~nm}$. Photogrammetric Engineering \& Remote Sensing, v. 62, n. 2, p. 195-200.

GITELSON, A. 1992. The peak near $700 \mathrm{~nm}$ on radiance spectra of algae and water: relationships of its magnitude and position with chlorophyll concentration. International Journal of Remote Sensing, v. 13 , n.17, p. $3367-3373$.

CICERELLI, R.E.; GALO, M.L. B.T. 2015. Sensoriamento remoto multifonte aplicado na detecção do fitoplâncton em águas interiores. Revista Brasileira de Engenharia Agrícola e Ambiental, [s.1.], v. 19, n. 3, p.259-265. http://dx.doi.org/10.1590/1807-1929/agriambi.v19n3p259-265.

FERREIRA, M. S.; GALO, M. L. B. T.. 2013. Chlorophyll a spational inference using artificial neural network from multispectral imagens and in situ measurements. Anais da Academia Brasileira de Ciências, v.85, p.519-532. 